\title{
Review
}

\section{Unsolved mysteries of intestinal $M$ cells}

\begin{abstract}
Summary
$M$ cells are highly specialised cells present within the epithelium overlying organised lymphoid follicles of the small and large intestine. They play a central role in the initiation of mucosal immune responses by transporting antigens and microorganisms to the underlying lymphoid tissue. In this way the mucosal immune system encounters the limitless variety of antigens that enter the body through the gut mucosa and reacts by mounting specific mucosal and systemic immune responses.

Despite the role of $M$ cells in mucosal defence many basic aspects of their biology, the most controversial being their origin within the follicle associated epithelium (FAE), still remain the subjects of debate. Recently, new information on the complex interactions of luminal microorganisms, mucosal immune system, and epithelial cells, that are instrumental in the induction of this cell phenotype, have become available. Here, the most novel data and hypotheses on $\mathrm{M}$ cell genesis and function in the gut are reviewed and discussed.
\end{abstract}

\section{Introduction}

The main task of the epithelium overlying mucosal surfaces of the intestinal tract is to provide an effective barrier to the vast majority of macromolecules and microorganisms present in the intestinal lumen. This is achieved by several means. Firstly, the epithelium is formed by cells joined by tight junctions that allow passage of water and ions but provide an effective mechanical barrier to macromolecules. ${ }^{12}$ Secondly, mucosal surfaces are covered by local secretions of mucus, secretory IgA antibodies, and by a thick glycocalix..$^{3-6}$ These features and the closely packed carpet of microvilli present on absorptive cells prevent contact and binding of macromolecules and potential pathogens to the epithelium. On the other hand, the intestinal epithelium must also provide portals through which antigens and microorganisms are delivered to the intestinal immune system in order to induce immune responses. In fact, it is now established that antigenic penetration of epithelial barriers is the first critical step in the generation of protective mucosal and systemic immune responses. ${ }^{7}$ The ability of the intestinal epithelium to transport antigens and microorganisms is strategically restricted to the $\mathrm{FAE}^{8}$ that overlies the organised mucosal associated lymphoid tissue in the gut. ${ }^{9}$ To accomplish this task, the FAE has evolved features that distinguish it from the surrounding absorptive epithelium. The most remarkable adaptation is the presence of a relatively small number of highly specialised antigen sampling membranous $(\mathrm{M})$ cells. $^{10}$

\section{Features of intestinal $M$ cells}

MORPHOLOGY AND FUNCTION

$M$ cells were first observed by transmission electron microscopy in rabbit appendix. ${ }^{11}$ They display distinctive morphological features that distinguish them from surrounding enterocytes. The brush border is poorly organised with short irregular microvilli, and the thick glycocalix usually associated with absorptive cells is absent. ${ }^{12}{ }^{13}$ These adaptations allow material in the intestinal lumen to have easy access to the apical domain of $M$ cells where it is internalised and then transported to the underlying lymphoid tissue. The mechanisms by which $\mathrm{M}$ cells take up microorganisms and macromolecules vary according to the nature of this material. Large particles and bacteria induce phagocytosis, which is associated with ruffling of the apical plasma membrane of the $M$ cell and rearrangement of the actin cytoskeleton, which permits active formation of pseudopod-like structures. ${ }^{14}{ }^{15}$ Viruses and other adherent particles are taken up by endocytosis via clathrin coated vesicles, ${ }^{16}$ whereas non-adherent material is internalised by fluid phase endocytosis. ${ }^{11}{ }^{17}$ In any case, internalisation is quickly followed by transport of endocytotic vesicles to the endosomal compartment and then by exocytosis to the basolateral membrane. The biochemical events involved in the intracellular transport of endocytotic vesicles in $\mathrm{M}$ cells have not been thoroughly explored but it appears that this is regulated in the same manner as polarised transport observed in other epithelial cells. ${ }^{18} 19$

A typical feature of $\mathrm{M}$ cells is that, unlike other intestinal epithelial cells, the basolateral surface is deeply invaginated to form intraepithelial pockets that are in intimate contact with specialised lymphocytes that migrate to this peculiar compartment from lymphoid tissue ${ }^{20}$ (fig $1 \mathrm{~A}, \mathrm{~B}$ ). This modification of the basolateral domain of $M$ cells is believed to be a way of shortening the distance that endocytotic vesicles have to travel to reach immunocompetent areas. ${ }^{1622}$ Thus $\mathrm{M}$ cell pockets provide the first opportunity for contact between antigens, penetrating the epithelial barrier and specialised immune cells. It has been proposed that $M$ cell pockets are the sites where intraepithelial lymphocytes (IEL) can interact early with internalised antigens in an environment sequestered from regulatory elements of the mucosal immune system. ${ }^{22}$

SIMPLY ANTIGEN SAMPLING CELLS OR SOMETHING MORE? The phenotype of IEL residing in $M$ cell pockets has been analysed in Peyer's patch tissue of humans and other species. ${ }^{23-25}$ Although there is remarkable variation between animal species, most of the $M$ cell associated IEL are $\alpha / \beta$ memory $T$ cells. In the rabbit a distinctive phenotype lacking both CD 4 and CD8 was also observed. ${ }^{26} \mathrm{~T}$ cells in the pockets are often associated with naive $\operatorname{IgM}^{+}$and $\mathrm{IgD}^{+} \mathrm{B}$ cells and macrophages, whereas $\operatorname{IgG}^{+}$and $\operatorname{IgA}^{+}$cells have rarely been observed. ${ }^{27}$

The lymphoid cells harboured within $M$ cell pockets represent all the cell types required to initiate a specific immune response but their true functional significance remains unclear. An immunological role for the $M$ cell associated lymphocytes is suggested by the observation that their number rapidly increased after application of a bacterial challenge..$^{1528}$ In these circumstances, a large number of cells migrate into the intestinal lumen via $M$ cell gated diapedesis. ${ }^{28}$ The physiological relevance of $M$ cell regulated passage of lymphocytes into the gut lumen and their role in this location remain to be determined.

A broadly accepted view is that $M$ cells are simply conduits through which antigens reach the immunocompetent areas of the gut associated lymphoid tissue. According to some authors $\mathrm{M}$ cells are provided with the cytoplasmic

Abbreviations: FAE, follicle associated epithelium; IEL, intraepithelial lymphocytes. 
A
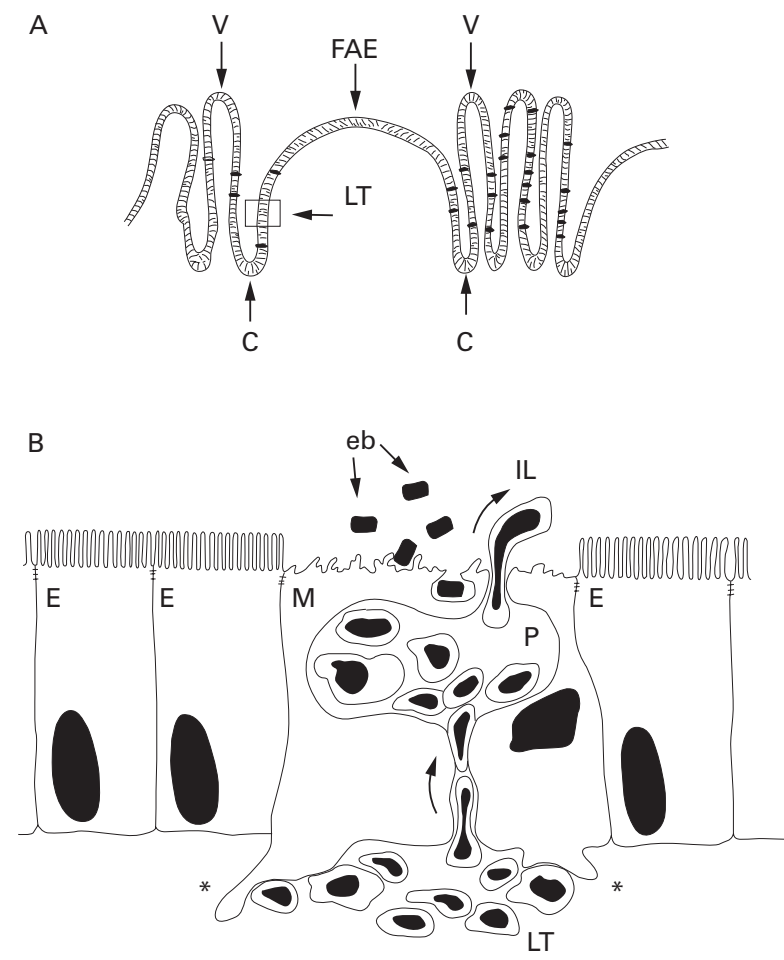

Figure 1 In Peyer's patches, the lymphoid tissue (LT) is separated from the intestinal lumen (IL) by the follicle associated epithelium (FAE) (A). The cells forming the FAE originate in the closely associated crypts $(C)$ and migrate upwards to the apical region of the dome. Lymphoid follicles are embedded within villi (V). Part of the FAE is indicated in the box (arrowhead) and enlarged (B) to show a typical $M$ cell $(M)$ with adjacent enterocytes $(E)$. The basal membrane of the $M$ cells deeply invaginates forming cytoplasmic pockets $(P)$ harbouring lymphoid cells migrating into them from the lymphoid tissue. $M$ cells also possess basal processes that deeply protrude into the lymphoid tissue (asterisks) where they make contact with cells of the immune system. Enterobacteria (eb) and other antigens adhere to the apical area of the $M$ cells and are subsequently internalised and transported to the mucosal immune system. $M$ cells are also a migration route for lymphocytes moving into the intestinal lumen. The function of these intraluminal cells, the number of which markedly increases after bacterial challenge, remains unknown.

components required to process antigens, ${ }^{29}$ including cathepsin $\mathrm{E}^{30}$ an aspartic proteinase involved in antigen processing by a $\mathrm{B}$ cell lymphoma. ${ }^{31}$ It is notable that expression of major histocompatibility complex class II molecules has been reported on the basolateral domain facing the pockets. ${ }^{27}{ }^{29} \mathrm{M}$ cells may also have the potential to participate actively in the generation of mucosal immune responses by signalling to lymphocyte by releasing IL- $1 .^{32}$ In addition, $M$ cells possess basal processes that deeply protrude into the lymphoid tissue where they make contact with cells of the immune system. ${ }^{33}$ Taken together these data would suggest a more active and complex role of intestinal $\mathrm{M}$ cells in the early phases of mucosal immune responses.

HETEROGENEITY OF M CELL POPULATION

$M$ cells were discovered more than 25 years ago ${ }^{11}$ but despite intensive investigations a clear cut $\mathrm{M}$ cell marker, independent of species and location in the gut, has not been found. For example, in the rabbit, but not in other species so far tested, $M$ cells express the intermediate filament protein vimentin ${ }^{34-36}$ that is typical of cells of mesenchymal origin. In the past, $M$ cells have been mainly identified by the absence of hydrolytic enzymes, such as alkaline phosphatase, that are abundant in the brush border of enterocytes. ${ }^{37-39}$ However, the reliability of alkaline phosphatase as a specific negative marker has been questioned by several authors on the basis of the extreme variability in the content of this enzyme in all FAE cells. ${ }^{18}{ }^{40}$ More recently it has been reported that mouse $M$ cells display a different distribution of the actin associated protein villin. ${ }^{41}$ This is restricted to the apical region of enterocytes but in $M$ cells it is diffusely distributed in the cytoplasm reflecting a differentially organised cytoskeleton and probably a decreased structural rigidity.

The heterogeneity of $M$ cell populations is also well exemplified by the highly differentiated expression of polysaccharides of membrane bound glycoproteins and glycolipids. The use of a large panel of lectins to stain FAE cells has revealed that in some cases $M$ cells display a different glycosylation state compared with neighbouring enterocytes. ${ }^{42} 43$ In mice, for example, small intestine Peyer's patch $M$ cells predominantly express $\alpha(1-2)$-fucose that can be detected by Uleus europeaus (UEA-1) lectin. ${ }^{33} 4{ }^{44}$ The pattern is different in caecal patches where $M$ cells are stained by the same lectin but also express other terminal saccharides. ${ }^{42}{ }^{45}$ In humans, the glycosylation pattern is different from that of other species as $M$ cells preferentially display the sialyl Lewis A antigen. ${ }^{46}$

Membrane bound glycoconjugates are believed to play an important role in microbial-M cell interactions. ${ }^{47}$ In the light of this consideration the extreme variability of these molecules on the $\mathrm{M}$ cell surface has been interpreted as an effective way to generate a broad repertoire of $M$ cell surface binding molecules to recognise bacteria borne lectins. ${ }^{22} 47$

$M$ cell specific glycoconjugates have been successfully used as targets for oral and nasal delivery of antigens and particulate carrier. ${ }^{48}{ }^{49}$ However, regional differences in the glycosylation pattern of $M$ cells could influence the outcome of an antigen challenge. Studies in sheep have shown that the location in the gut in which antigen transport takes place may have profound effects on the immune responses. ${ }^{50}$ Both systemic and mucosal immune responses were observed when the vaccine vector was delivered to jejunal, but not ileal, Peyer's patches. In the latter case only a systemic response was observed.

M CELLS AS THE MAIN PORTAL OF ENTRY FOR ENTEROPATHOGENS

In contrast with the positive role of $\mathrm{M}$ cells in the initiation of mucosal immune responses, their capacity for antigen sampling can facilitate invasion by potential harmful intestinal microorganisms. ${ }^{51}$ The risk is mitigated by the fact that these pathogens are directly delivered to areas of the immune system fully equipped to cope with such an emergency. Nevertheless, some microorganisms exploit $\mathrm{M}$ cells as an entry site to breach the mucosal barrier and establish local and systemic infections. ${ }^{52} 53$ The most dramatic evidence of such ability is provided by studies performed on Salmonella, ${ }^{54}{ }^{55}$ Shigella, ${ }^{56}$ and Yersinia ${ }^{57}$ in different animal models. A detailed analysis of the various mechanisms used by these bacteria is beyond the scope of this article but has recently been reviewed elsewhere. ${ }^{58}$

\section{Origin and fate of intestinal $M$ cells: facts and hypotheses}

Intestinal epithelial cell maturation and differentiation is a well known phenomenon which is completed in a geographically well organised migration. ${ }^{59-61}$ The epithelium of each follicle derives from surrounding crypts, each crypt being a clonal unit, ${ }^{62}{ }^{63}$ which are characterised by two distinct axes of migration and differentiation. Cells located on the villus side of the crypt differentiate into absorptive enterocytes, goblet, and enteroendocrine cells. Cells on the FAE side of the crypt move onto the dome, acquiring features of follicle associated enterocytes and $\mathbf{M}$ cells. ${ }^{7}$ The final differentiation of intestinal epithelial cells 
takes place as they migrate in vertical bands to the apical extrusion zone of the villus and FAE. ${ }^{59}$ Within this scenario two different theories on $\mathrm{M}$ cell genesis in the FAE have been formulated. Although it is accepted that enterocytes and $M$ cells have a common precursor it has been postulated that $\mathrm{M}$ cells may originate in the crypts as a distinct cell lineage from stem cells via an independent differentiation programme or from enterocytes on interaction with the local lymphoid microenvironment.

Initially, the hypothesis that $M$ cells are derived from undifferentiated crypt cells was based on ultrastructural studies and on the utilisation of ${ }^{3} \mathrm{H}$ thymidine as a proliferation marker. ${ }^{3} \mathrm{H}$ thymidine labelled cells were observed in crypts adjacent to the dome ${ }^{13}$ and within 24 hours they migrated at the dome periphery where they acquired morphological and structural features of $M$ cells. ${ }^{13}{ }^{64}$ The most recent experimental evidence supporting this idea mostly comes from studies on differential expression of glycoconjugates on $\mathrm{M}$ cell membranes. It has been demonstrated, in both the rabbit and mouse, that lectin labelled $M$ cells were not restricted to the dome of the FAE but were also detected in crypts. ${ }^{33}{ }^{65}$ These data indicated that a subpopulation of crypt cells is predetermined as $M$ cells before attaining their morphological and functional features. More recently another morphological and histochemical study has provided further evidence that supports this view. ${ }^{66}$ Here it was also determined that the random distribution of the sites where lymphocytes invade the FAE did not correlate with the organisation of $M$ cells in radial strips that emerged from the dome associated crypts. These data led the authors to conclude that $M$ cell formation is restricted in specialised dome associated crypts and that FAE associated enterocytes do not have the potential to convert into $M$ cells.

In contrast, data from other groups point in a different direction. Originally, lymphocyte induced $M$ cell formation was proposed by Smith and colleagues. ${ }^{39}{ }^{67}$ Recently, an elegant in vitro co-culture system has been established in which murine Peyer's patch derived lymphocytes convert human intestinal Caco2 cells into functional $\mathrm{M}$ cells. ${ }^{68}$ Although it has been highlighted that due to the lack of terminal differentiation it is difficult to consider these adenocarcinoma cells as differentiated enterocytes, ${ }^{69}$ this model provides clear cut demonstration that lymphoepithelial cross talk plays a central role in controlling the genesis of differentially specialised epithelial cell populations. In addition, in vivo studies have provided evidence that bone marrow transplantation in SCID syngeneic mice induced the formation of $\mathrm{M}$ cells, thereby demonstrating that $\mathrm{M}$ cell genesis is immunoregulated..$^{68}$ Furthermore, our group has reported that short term exposure (1-3 hours) to a non-intestinal bacterium induced dramatic alterations of the FAE that included a marked increase in the number of fully operational $M$ cells in topographically restricted areas of the FAE. ${ }^{15}{ }^{71} 72$ In this case the brevity of the experimental procedure demonstrated that the appearance of $M$ cells, under certain experimental conditions, can take place in time scales too short to be explained by migration of $\mathrm{M}$ cells originated in crypts. ${ }^{22}$

Is it possible, in the light of these apparently conflicting data, to reconcile the two theories? One possibility is that the rapid increase in $M$ cells after microbial challenge could be explained as short term differentiation of predetermined $\mathrm{M}$ cells. ${ }^{65}$ According to this hypothesis, these immature pre- $M$ cells would elude detection because of lack of well defined ultrastructural and histochemical markers and certain antigenic challenges would provide the ultimate stimulus to drive their differentiation into mature operational M cells. ${ }^{65}$ The presence of cells within the FAE with the ability of rapidly becoming antigen sampling cells would explain the changes in the FAE following bacterial challenge. It also might be interpreted as an emergency system to boost immune responses by increasing the amount of antigenic substrate in the inductive sites of the mucosal immune system. ${ }^{71}{ }^{72}$

The theory of lymphocyte induced $\mathrm{M}$ cell formation raises a question. How can lymphoid cells transform an enterocyte into an $M$ cell? Indeed, while the genesis of $M$ cells from undifferentiated crypt cells reflects the conventional differentiation pathway of other intestinal epithelial cells, the mechanism by which lymphocytes would induce the $M$ cell phenotype remains a matter of speculation. The $\mathrm{Caco} 2 \mathrm{co}$-culture system has provided clear evidence that $\mathrm{B}$ cells play a major role in the conversion of enterocytes. ${ }^{68}$ More recently, the role of B cells in the formation of both FAE and $M$ cells has been confirmed by in vivo experiments involving B cell knockout mice. ${ }^{73}$ At this time, however, the biochemical and molecular events involved are unknown. It was proposed that lymphocytes must deliver two different signals to enterocytes, one with the ability to disassemble the brush border and the second to trigger transcytosis. ${ }^{68}$ This hypothesis was based on the observation that the sole disorganisation of the brush border in Caco 2 cells, via suppression of villin by specific antisense RNA, ${ }^{68} 74$ was not sufficient to trigger transcytosis. Nevertheless, cells with morphological and functional features of $\mathrm{M}$ cells that were not in contact with lymphocytes were observed in other experimental systems. ${ }^{72}{ }^{75}$ In one such case ${ }^{72}$ rapid appearance of operational $M$ cells after bacterial challenge in vivo was preceded by an increase in the number of cells displaying intermediate phenotype between enterocytes and $M$ cells. Most of these cells, probably transitional stages, did not harbor lymphocytes, thereby suggesting that under certain conditions the stimulus provided only by a soluble molecule(s) may suffice to induce the $M$ cell phenotype without the need for physical interactions with lymphocytes.

Interestingly, $M$ cells are characterised by their peculiar distribution in the FAE. They are relatively abundant in the periphery but totally absent at the apical region. ${ }^{13}{ }^{72}$

The observation that $M$ cells are never observed in the apical region of the FAE poses a new question. What is their fate? In view of the highly dynamic nature of the FAE it has been hypothesised that $M$ cells may revert back to enterocytes as they reach the apical area of the FAE. ${ }^{41}$ This is also supported by the observation that no apoptotic cells were found at the sites where $M$ cells are located ${ }^{76}$ thereby suggesting that $M$ cells are not preferentially extruded at the periphery. Thus $M$ cells may represent a transient and reversible phenotype.

The situation appears to be different in the rabbit model. Rabbit $M$ cells have the advantage of expressing the intermediate filament vimentin ${ }^{34-36}$ and are easily identified within the FAE. Consistent with the distribution of $M$ cells already observed in other animal species, vimentin positive cells are restricted at the periphery of FAE and are totally absent at the apical area. ${ }^{72}$ Immunohistochemical analysis of the rabbit FAE revealed the presence of $M$ cell debris in the lumen over the dome shoulders, where desquamation of $\mathrm{M}$ cells was also observed. ${ }^{77}$ This strongly suggests that $M$ cells are extruded at the level of the shoulders. Furthermore, if they were to revert back to enterocytes as they migrate towards the apex of the dome, one would expect to observe an intermediate phenotype in the close vicinity of the apical, $M$ cell free area. Instead, vimentin positive cells stop abruptly at the dome shoulders ${ }^{72}$ suggesting that, at least in the rabbit model, $M$ cells are probably extruded at the periphery rather than at the top of the dome. 


\section{Concluding remarks}

$\mathrm{M}$ cells are important in regulating access of microorganisms and antigens to areas of the intestinal immune system equipped to generate protective immune responses. However, much remains to be learnt of the biology of this cell. The importance of finding the best way to induce protective mucosal immune responses is highlighted by the accepted view that the systemic immune response is not adequate to fight the vast majority of pathogens to which we are exposed throughout life. It is believed that over $95 \%$ of human pathogenic microorganisms target host cells after crossing epithelial barriers. For this reason it would be highly desirable to induce specific immunity at the site of invasion. The need to potentiate immunological defence at the mucosal level is further stressed by the fact that in adult individuals the area of the mucosal surfaces reaches the impressive size of $400 \mathrm{~m}^{2}{ }^{78} \mathrm{~A}$ better understanding of the mechanisms involved in the recognition and transepithelial transport of antigens, along with the molecular basis of $M$ cell formation within the FAE, will represent an important step forward in the design of new strategies to improve oral delivery of biologically active compounds to the intestinal immune system.

C NICOLETTI

Laboratory of Gut Immunology,

Diet Health and Consumer Sciences Division,

Institute of Food Research, Norwich Research Park, Colney, Norwich NR4 7UA, UK

claudio.nicoletti@bbsrc.ec.uk

I am indebted to M J Taussig for his friendly and valuable support. I also wish to thank P J Kilshaw and J P Kraehenbuhl for helpful comments and discussions. The work in our laboratory has been funded by the Ministry of Agriculture Food and Fisheries of the United Kingdom and by the Biotechnology and Biological Sciences Research Council.

1 Madara JL, Nash S, Moore R, et al. Structure and function of the intestinal epithelial barrier in health and disease. Monogr Pathol 1990;31:306-24

2 Bjarnason I, Mac Pherson, Hollander D. Intestinal permeability. Gastroenterology 1995;108:1566-81.

3 Mc Ghee JR, Kiyono H. New perspective in vaccine development: mucosal immunity to infections. Infect Agents Dis 1993;2:55-73.

4 Frey A, Giannasca KT, Weltzin R, et al. Role of glycocalix in regulating access to plasma membranes of intestinal epithelial cell: implications for microbial attachment and oral vaccine targeting. F Exp Med 1996;1996: $1045-59$

5 Kett K, Baklien K, Bakken A, et al. Intestinal B cell isotype response in relation to local bacteria load: evidence for IgA subclass adaptation. Gastroention to local bacteria load:

6 Maury J, Nicoletti C, Guzzo-Chambraud F, et al. The filamentous brush border glycocalix, a mucine-like marker of enterocytes hyper polarization. border glycocalix, a mucine-like
Eur f Biochem 1995;228:323-31.

7 Neutra MR, Pringault R, Kraehenbuhl JP. Antigen sampling across epitheial barriers. Annu Rev Immunol 1996;14:275-300.

8 Allan $\mathrm{CH}$, Trier JS. Structure and permeability differ in subepithelial villus and Peyer's patches follicles capillaries. Gastroenterology 1991;100:1172-9.

Hein WR. Organizanion of mucosal lymphoid tissue. Curr Top Microbiol Immunol 1999;236:1-15.

10 Owen RL, Jones AL. Epithelial cell specialization within human Peyer's patch: An ultrastructural study of intestinal lymphoid follicle. Gastroenetrology 1974;66:189-203.

11 Bockamn DE, Cooper MD. Pinocytosis by epithelium associated with lymphoid follicles in the bursa of Fabricius, appendix and Peyer's patches. An electron microscopy study. Am f Anat 1973;136:455-78.

12 Bockman DE. Functional histology of appendix. Arch Histol Cytol 1983;46: $271-92$.

13 Bye WA, Allan CH, Trier JS. Structure, organization and distribution of $M$ cells in Peyer's patches. Gastroenterology 1984;86:789-801.

14 Fujimura Y. Functional morphology of microfold cells (M) in Peyer's patches. Phagocytosis and transport of BCG by M cells into rabbit Peyer's patches. Phagocytosis and transport of BCG

15 Borghesi C, Bertelli E, Regoli M, et al Modifications of the FAE by short term exposure to a nonintestinal bacterium. F Pathol 1996;180:326-32.

16 Neutra MR, Phillips TL, Mayer EL, et al. Transport of membrane-bound macromolecules by M cells in the FAE of rabbit Peyer's patches. Cell Tissue Res 1987;247:536-46.

17 Owen RL. Sequential uptake of horseradish peroxidase by lymphoid follicle epithelium of Peyer's patches in normal unobstructed mouse intestine: an ultrastructural study. Gastroenterology 1977;72:440-51.

18 Gebert A. The role of $\mathrm{M}$ cells in the protection of mucosal membrane. Histochem Cell Biol 1997;108:455-70.

19 Druben DG, Nelson WJ. Origin of cells polarity. Cell 1996;84:335-44.

20 Wolf JL, Bye WA. The membranous (M) epithelial cells and the mucosal immune system. Аnпи Rev Med 1984;35:95-112.

21 Regoli M, Bertelli E, Borghesi C, et al. Three (3D)-reconstruction of $M$ cells in rabbit Peyer's patches: definition of intraepithelial compartment of the in rabbit Peyer's patches: definition of intraepithelial con

22 Neutra MR. Current concepts in mucosal immunity. V. Role of $M$ cells in transepithelial transport of antigens and pathogens to the intestinal immune system. Am f Physiol 1998;274:G785-91.
23 Farstad IN, Halstensen T, Fausa S, et al. Heterogeneity of M cells-associated $\mathrm{B}$ and T cells in human Peyer's patches. Immunology 1994;83:921-37.

24 Spencer J, Finn T, Isaacson PG. Human Pever's patches: an immunohistochemical study. Gut 1986;27:405-10.

25 Ermak TH, Owen RL. Differential distribution of lymphocytes and accessory cells in mouse Peyer's patches. Anat Rec 1986;215:144-52.

26 Ermak TH, Steger HJ, Pappo J. Phenotypically distinct subpopulation of T cells in domes and $M$ cells pockets of rabbit gut-associated lymphoid tissue. Immunology 1990;71:530-7.

27 Jarry AM, Robaszkiewicz M, Brousse N, et al. Immune cells associated with $\mathrm{M}$ cells in the follicle-associated epithelium of Peyer's patches in the rat. Cell Tissue Res 1989;255:293-8.

28 Regoli M, Borghesi C, Bertelli E, et al. A morphological analysis of lymphocyte traffic in Peyer's patches after an in vivo antigenic stimulation. Anat Rec 1994:239:47-54.

29 Allan CH, Mendrick PL, Trier JS. Rat intestinal M cells contain acidic endosomal-lysosomal compartments and express class II major histocomendosomal-lysosomal compartments and express class II major histocor
patibility complex determinants. Gastroenterology 1993;104:698-708.

30 Finzi G, Carnaggia M, Copella C, et al. Cathepsin E in follicle-associated epithelium of intestine and tonsils: localization to $M$ cells and possible role in antigen processing. Histochemistry 1993;99:201-11.

31 Nagura $\mathrm{H}$, Othani $\mathrm{H}$, Masuda T, et al. HLA-DR expression of $M$ cells overlying Peyer's patches is a common feature of human small intestine. Acta Pathol fpn 1991;41:818-23.

32 Pappo J, Mahlman RT. Follicle epithelial M cells are a source of IL-1 in Peyer's patches. Immunology 1993;78:505-7.

33 Giannasca PJ, Giannsca KT, Falk P, et al. Regional differences in glycoconjugates of intestinal $\mathrm{M}$ cells in mice: potential targets for mucosal vaccine. Am F Physiol 1994;267: G1108-21.

34 Jepson MA, Mason CM, Bennett MK, et al. Co-expression of vimentin and cytokeratins in $M$ cells of rabbit intestinal lymphoid follicle associated epithelium. Histochem f 1992;24:33-9.

35 Gebert A, Hach G, Bartels H. Co-localization of vimentin and cytokeratins in $M$ cells of rabbit gut associated lymphoid tissue. Cell Tissue Res 1992;269:331-40.

36 Gebert A. Identification of $M$ cells in the rabbit tonsils by vimentin immunohistochemistry and in vivo protein transport. Histochem Cell Biol 1995;104:211-20.

37 Owen RL, Bhalla DK. Cytochemical analysis of alkaline phospatase and esterase activity and of lectin-binding and anionic sites in rat and mouse Peyer's patch M cells. Am f Anat 1983;168:199-212.

38 Smith MW, James PS, Tivey DR, et al. Automated histochemical analysis of cells populations in the intact follicle-associated epithelium of the mouse Peyer's patch. Histochem $\mathcal{F}$ 1988;20:443-8.

39 Smith MW, James PS, Tivey DR. M cells number increase after transfer of SPF mice to a normal house environment. Am f Pathol 1987;128:385-9.

40 Jepson MA, Simmons NL, Savidge TC, et al. Selective binding and transcytosis latex microspheres by rabbit M cells. Cell Tissue Res 1993;271:399-405.

41 Kerneis S, Bogdanova A, Colucci-Guyon E, et al. Cytosolic distribution of villi in $M$ cells from mouse Peyer's patches correlates with the absence of a vrush border. Gastroenterology 1996;110:515-21.

42 Gebert A, Hach G. Differential binding of lectins to $M$ cells and enterocytes in the rabbit caecum. Gastroenterology 1993;105:1350-61.

43 Clark MA, Jepson MA, Simmons NL, et al. Differential expression of lectin binding sites defines mouse intestinal $M$ cells. $\mathcal{F}$ Histochem Cytochem 1993; 41:1679-87.

44 Falk P, Roth KA, Gordon JI. Lectins are sensitive tools for defining the differentiation programs of epithelial cell lineages in the developing and adult mouse gastrointestinal tract. Am f Physiol 1994;266:G987-1003.

45 Clark MA, Jepson MA, Simmons NL, et al. Differential surface characteristics of $M$ cells from mouse intestinal Peyer's patches and caecal patches. Histochem f 1994;26:271-80.

46 Giannasca PJ, Giannasca KT, Leichtner AM, et al. Human intestinal M cells display the sialyl Lewis A antigen. Infect Immun 1999;67:946-53.

47 Neutra MR, Giannasca PJ, Giannasca KT, et al. M cells and microbial pathogens. In: Blaser M, Smith PD, Ravodin JI, et al, eds. Infections of the GI tract. New York: Raven, 1995:163-78.

48 Giannasca PJ, Boden JA, Monath TP. Targeted delivery of antigens to hamster nasal lymphoid tissue with M cells directed lectins. Infect Immun 1997; $65: 4288-98$.

49 Foster N, Clark MA, Jepson MA, et al. Uleus europeaus 1 lectin targets micropheres to mouse Peyer's patch M cells in vivo. Vaccine 1998;16:536-41.

50 Mutwiri G, Watts F, Lew L, et al. Ileal and jejeunal Peyer's patches play distinct roles in mucosal immunity of sheep. Immunology 1999;97:455-61

51 Neutra MR, Frey A, Kraehenbuhl JP. Epithelial M cells: gateways for mucosal infection and immunization. Cell 1996;86:345-8.

52 Jepson MA, Clark MA. Studying M cells and their role in infections. Trends Microbiol 1998;6:359-65.

53 Fujimura Y, Owen RL. M cells as portals of infection: clinical and pathophysiological aspects. Infect Agents Dis 1996;5:144-56.

54 Kohobata S, Yokobata H, Yabuuchi E. Cytopathogenic effect of Salmonella typhi GIFU 10007 on M cells of murine ileal Peyer's patches in ligated ileal loops: an ultrastructural study. Microbiol Immunol 1986;30:1225-37.

55 Jones BD, Ghori N, Falkow S. Salmonella typhimurium initiates murine infection by penetrating and destroying the specialized epithelial $M$ cells of infection by penetrating and destroying the specializ
the Peyer's patches. F Exp Med 1994;180:15-23.

56 Perdomo JL, Cavaillon JM, Huerre M, et al. Acute inflammation causes epithelial invasion and mucosal destruction in experimental shigellosis. $\mathcal{f}$ Exp Med 1994:180:1307-19.

57 Grutzkau A, Hansky C, Hahn H, et al. Involvement of M cells in the bacterial invasion of Peyer's patch: a common mechanism shared by Yersinia enterocolitica and other enteroinvasive bacteria. Gut 1990;3:1011-15.

58 Sansonetti PJ, Phalipon A. M cells as portals of entry for enteroinvasive pathogens: mechanisms of interaction, consequences for the disease process. Semin Immunol 1999;11:193-203.

59 Hermiston M, Green RP, Gordon JI. Chimeric-transgenic mice represent a powerful tool for studying how the proliferation and differentiation programs of intestinal epithelial cell lineages are regulated. Proc Natl Acad Sci USA 1993;90:8866-70.

60 Heath JP. Epithelial cell migration in the intestine. Cell Biol Int 1996;20:139-46.

61 Louvard D, Kedinger M, Hauri HP. The differentiating intestinal epithelial cells: establishment and maintenance of functions through interactions between cellular structures. Annu Rev Cell Biol 1992;8:157-95. 
62 Ponder BAJ, Schmidt GH, Wilkinson MM, et al. Derivation of mouse intestinal crypts from single progenitor cells. Nature 1985;313:689-91.

63 Rubin DC, Ong D, Gordon JI Cellular differentiation in the emerging fetal rat small intestinal epithelium: mosaic pattern of gene expression. Proc Natl Acad Sci USA 1989;86:1278-82.

64 Bhalla DK, Owen RL. Cell renewal and migration in lymphoid folicle of Peyer's patches and caecum. An autoradiographic study in mice. Gastroenterology 1982;82:232-42.

65 Gebert A, Posselt W. Glyconjugate expression defines the origin and differentiation pathway of intestinal M cells. F Histochem Cytochem 1997;45: 1341-50.

66 Gebert A, Fassbender S, Werner K, et al. The development of $M$ cells in Peyer's patches is restricted to specialized dome-associated crypts. Am $\mathcal{F}$ Pathol 1999;154:1573-82.

67 Smith MW, Peacock MA. Lymphocyte induced formation of antigen transporting $M$ cells from fully differentiated mouse enterocytes. In: Robinson JWL, ed. Mechanisms of intestinal adaptation. Lancaster: MTP, 1982:573-83.

68 Kerneis S, Bogdanova A, Kraehenbuhl JP, et al. Conversion by Peyer's patch lymphocytes of human enterocytes into M cells that transport bacteria. Scilymphocytes of human

69 Owen RL. Mid-life crysis for M cells. Gut 1998;42:11-12.

70 Sharma R, Schumacher U, Adam E. Lectin histochemistry revealed the mal bone marrow transplantation. $\mathcal{F}$ Histochem Cytochem 1998;46:143-8.
71 Meynell HM, Thomas NW, James PS, et al. Upregulation of microsphere transport across the follicle-associated epithelium of Peyer's patch by exposure to S. pneumoniae R36a. FASEB F 1999;13:611-19.

72 Borghesi C, Taussig MJ, Nicoletti C. Rapid appearance of M cells after microbial challenge is restricted at the periphery of the follicle-associated epithelium of Peyer's patch. Lab Invest 1999;79:1391-401.

73 Golovkina TV, Schlomchik M, Hannum L, et al. Organogenic role of B lymphocytes in mucosal immunity. Science 1999;286:1965-8.

74 Costa de Beauregard NA, Pringault E, Robine S, et al. Suppresion of villin by antisense RNA impairs brush border assembly in polarized epithelial intestinal cells. EMBO ₹ 1995;14:409-21.

75 Ermak TH, Steger HJ, Strober S, et al. M cell and granular mononuclear cells in Peyer's patch domes of mice depleted of their lymphocytes by total lymphoid irradiation. Am f Pathol 1989;134:529-37.

76 Debard N, Sierro F, Kraehenbuhl JP. Development of Peyer' patches, follicle-associated epithelium and $M$ cells. Lessons from immunodeficient and knockout mice. Semin Immunol 1999;11:183-91.

77 Owen RL, Piazza AJ. Where do $M$ cells go? Identification of their desquamation in rabbit Peyer's patch and appendix by immunohistochemical labeling of $M$ cells cytoplasmic vimentin (abstract). Gastroenterology 1998;114:A1057.

78 Brandtzaeg P, Backkevold ES, Fardstad IN, et al. Regional specialization in the mucosal immune system: what happens in the microcompartments? Immunol Today 1999;20:141-51. 